that the growth in demand in public markets sparks parallel growth in more lucrative markets such as the private markets of the developing countries. Manufacturer's interest in price tiering is conditional on a segmented market and public recognition that the lowest price is limited to a transparent selection of neediest countries. Evidence of industry support is illustrated by the current offer of a lowest tiered price for a "new" vaccine which is priced at $1 \mathrm{x}$ in industrial markets, $0.3-0.5 \mathrm{x}$ in the better off developing countries and offered at $0.1 \mathrm{x}$ to the defined set of poorest countries, in bands (Fig. 5).

Because of the 3-5 year lead times required to build and validate new production facilities, a manufacturer must make plant capacity decisions well in advance of real demand. The decision to invest in adequate capacity to serve the global markets is made on the credibility of demand estimates provided by the public sector. The more credible the public sector and the estimates, the more probable the investment. Currently, public sector credibility is quite low as policy makers have not been able to predict global demand or timing of national introduction and have not been able to secure the financing for the neediest countries. The public sector must provide far more accurate forecasts earlier in the lifecycle and must explore financial mechanisms to commit or guarantee the purchase of some quantity of product. If industry is convinced to make the marginal investment to serve global demand, rather than just in- dustrial country demand, the overall cost of production is reduced. More efficient management of costs will enable industry to provide vaccine for the neediest countries with the very lowest tiered price possible. In the absence of planning, future expansion of capacity to meet global demand is at best, slow and at worst, financially unjustifiable.

Finally, the public sector's capacity to centralize the procurement of vaccines for the neediest countries can provide an efficient mechanism to target the lowest tiered price to the neediest countries. By targeting procurement of new vaccines to obtain the lowest tiered price, the central procurement agency reinforces the system of price tiering and, through its own links with countries, reduces the transaction cost of providing the marginally priced vaccine. The public sector has the potential to leverage its current purchases of vaccines (through institutions such as UNICEF) to further strengthen relationships with key R\&D suppliers for earlier access to affordable, priority vaccines.

Health Specialist, The World Bank, Human Development Network 1818 H Street, NW

Washington, D.C. 20433

(formerly with Global Programme for Vaccines and Immunization (GPV), World Health Organization)

The author is indebted to Piers Whitehead, Mercer Management Consulting, London, UK for his analysis of the economics of the vaccine industry.

Anthony Fauci and Gregory Folkers (National Institute of Allergy and Infectious Diseases) discusses the role of US government agencies and the NIAID in particular, in the fight against infectious diseases and the part that vaccine research plays in this fight. From long term strategic decisions to specific research projects, Fauci illustrates the federal commitment to vaccine development and how it has and can impact both national and international health.

\title{
The role of US Government agencies in vaccine research and development
}

In this century, vaccines have without question been among our most powerful tools for preventing disease, disability and death and controlling health care costs. Recognizing the extraordinary benefits of vaccines, US government agencies charged with protecting and improving the public health have traditionally made vaccine research and development a top priority. Together with academic and industrial partners, government-supported scientists have helped develop many of our most useful vaccines, as well as several that will become available in the near future. These include new or improved vaccines that protect against rabies, Haemophilus influenza type $b$, pneumococcal pneumonia, pertussis, influenza, measles, mumps, rubella, hepatitis $A$ and $B$, typhoid fever and rotavirus ${ }^{1,2}$.

\section{IMAGE UNAVAILABLE FOR COPYRIGHT REASONS}

have facilitated some of the greatest successes in public health. Globally, smallpox has been eradicated, poliomyelitis S. FAUCI is on the verge of elimination, and other serious diseases such as measles have been dramatically reduced in many regions. Each year, vaccines against these three diseases alone save an estimated 7-8 million lives. By using measles vaccine in National Immunization Days and more widespread administration of currently available vaccines against rubella, hepatitis $\mathrm{B}$, and Haemophilus influenza type $\mathrm{b}$, another 3 million deaths could be prevented every year ${ }^{3}$ (see Bloom \& Widdus, page 480).

In the US, dramatic progress has been made against many serious diseases (Table). Age-appropriate vaccine coverage in the period June 1996 to June 1997 reached an all-time high, meeting or exceeding each of the targets set in President Clinton's 1993 Childhood Immunization Initiative ${ }^{4}$.

Fig. 1 Worldwide causes of death in 1996. (The World Health Report 1997, WHO) 
developments, infectious diseases remain the leading cause of death worldwide, and the third leading cause of mortality in the US. Of approximately 52 million deaths worldwide from all causes in 1996, more than 17 million were due to infectious diseases, including approximately 9 million among children (Fig. 1). In 1996 alone, an estimated 4.5-6 million deaths were attributed to two diseases, tuberculosis and malaria, for which no effective vaccines are available ${ }^{5}$. Vaccines also are lacking for many other serious infectious diseases that exact an enormous toll worldwide, such as sexually transmitted diseases (other than hepatitis B), respiratory syncytial virus and dysentery due to Shigella spp $^{2}$.

In addition to endemic diseases, we face the ongoing threat of new and re-emerging diseases and the widespread development of antimicrobial resistance, which underscore the need for a strong commitment to the development of new and improved vaccines. At least 30 newly recognized diseases and syndromes have been identified since 1980, including the acquired immunodeficiency syndrome (AIDS) and its etiologic agent, the human immunodeficiency virus (HIV). As a vivid reminder of the ever-present threat of disease emergence, the first known human cases of H5N1 avian influenza were identified in Hong Kong in 1997 (ref. 6). The multinational response to this outbreak has been extraordinary, including the US government's collaboration with industry in producing a recombinant vaccine for use in at-risk laboratory and health care personnel.

Resistance to antimicrobial agents has been observed in virtually all classes of organisms, resulting in a diminished capacity to treat many serious infections. For example, more than one-third of nosocomial Staphylococcus aureus infections in the US are resistant to methicillin, leaving vancomycin as the only reliable therapy ${ }^{7}$. We were given pause for thought in recent months when $S$. aureus isolates with increased resistance to vancomycin were identified for the first time in Japan and the US ${ }^{7,8}$. The threat of malaria also has been exacerbated by the widespread development of drug resistance. Among the 100 countries and territories where Plasmodium falciparum malaria is endemic, only Central America and Egypt have not recorded cases of chloroquine-resistance ${ }^{9}$. Resistance to fansidar and mefloquine is also common in many areas. As is the case with many diseases that disproportionately affect the developing world, an effective malaria vaccine is essential if this disease is to be controlled (see Miller \& Hoffman, page 520).

Collaborations and commitment

Most currently available vaccines, as well as those in

\begin{tabular}{|c|c|c|}
\hline \multicolumn{3}{|c|}{ The impact of vaccines in the United States } \\
\hline Disease & Peak incidence (year) & 1996 cases \\
\hline Diphtheria & $206,939(1921)$ & 2 \\
\hline Measles & $894,134(1941)$ & 508 \\
\hline Mumps & $152,209(1968)$ & 751 \\
\hline Pertussis & $265,269(1934)$ & 7,796 \\
\hline $\begin{array}{l}\text { Polio, paralytic } \\
\text { (wild poliovirus) }\end{array}$ & $21,269(1952)$ & 0 \\
\hline $\begin{array}{l}\text { Rubella } \\
\text { (German measles) }\end{array}$ & $57,686(1969)$ & 238 \\
\hline $\begin{array}{l}\text { Rubella, congenital } \\
\text { syndrome }\end{array}$ & $20,000(1964-5)$ & 4 \\
\hline
\end{tabular}

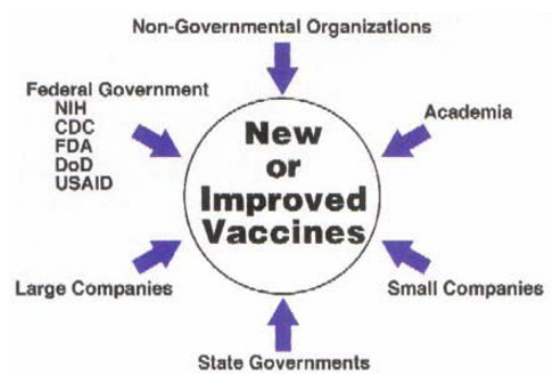

Fig. 2 Collaborative vaccine development. (Adapted from Pediatrics 100, 1015-1020; 1997)

the development "pipeline," have resulted from collaborations between partners in the public and private sector, including federal and state governments, small and large companies, academic research institutions and non-governmental organizations ${ }^{10-12}$ (Fig. 2).

The importance of vaccine development and the necessity for strong cross-sector partnerships has been recognized at the highest levels of government, both internationally and in the US. Last year, for example, the participants in the Denver Summit of Eight stressed their shared commitment to developing an HIV vaccine. Both Congress and President Clinton have made immunization, including vaccine research and development, a top priority, and the President has articulated the goal of developing an HIV vaccine within ten years. At the National Institutes of Health (NIH), funding for vaccine research and development has increased approximately 65 percent overall from fiscal year 1993 to fiscal year 1999 (ref. 13). NIH and other national research agencies are partners in the Children's Vaccine Initiative (CVI), launched in 1990 with the goal of developing safe, affordable and heatstable vaccines that can protect children against the infectious killers of childhood with a minimum of doses given orally early in life ${ }^{3}$. Congress has twice targeted funds in support of the CVI's work in facilitating the development, production, technology transfer, procurement and introduction of new vaccines. More recently, NIH helped establish the Multilateral Initiative on Malaria (MIM), an international consortium of government agencies, research organizations and donor agencies working to mobilize the scientific resources and political will needed to develop malaria control strategies and notably, a vaccin $\mathrm{e}^{14}$.

The private sector also has demonstrated a renewed commitment to vaccine development, thanks in part to revisions in product liability laws and new technology transfer legislation which have facilitated interactions between government, academia and industry ${ }^{15,16,17}$ (see Saldarini, page 485). Moreover, recent advances in gene cloning and expression, peptide synthesis and other technologies have created unprecedented opportunities for developing patentable "bioengineered" vaccines that promise a substantial return on research and development costs ${ }^{15}$.

\section{The Government players in vaccine research}

Within the federal government, more than 20 different agencies have a role in vaccine research ${ }^{10-12}$. Among these, the Centers for Disease Control and Prevention (CDC), the Department of Defense (DoD), the Food and Drug Administration (FDA), the United States Agency for International Development (USAID) 
and the NIH are the federal agencies with the largest investment in vaccine development ${ }^{10-12}$.

The roles of these different agencies in vaccine development are related and complementary, and span the spectrum from basic research to licensure and program implementation $^{10-12}$. For example, CDC conducts epidemiologic studies and surveillance needed to define health priorities, and develops recommendations for vaccine use through the Advisory Committee on Immunization Practices (ACIP). DoD performs research into vaccines that will protect against pathogens likely to be encountered by military personnel. USAID supports research on vaccines of particular relevance to young children in developing countries. The FDA establishes standards for the processes, facilities and pre- and post-licensing studies needed to insure the safety and effectiveness of vaccines. And the NIH supports much of the basic and clinical research in fields such as immunology and microbiology that leads to vaccine development. In addition, the NIH supports Vaccine Evaluation Units (VEV) and AIDS VEV's around the country, with broad capabilities to conduct prophylactic and therapeutic studies in children, adults and the elderly, as well as specific high-risk populations.

At the NIH, we have identified three broad goals in vaccine research:

- Identifying new vaccine candidates to prevent diseases for which no vaccines currently exist

- Improving the safety and efficacy of existing vaccines

- Designing novel vaccine approaches, such as new vectors and adjuvants.

The federal government also has an important coordinating function through the National Vaccine Program Office which is responsible for the coordination of government and nongovernment activities on research, licensing, production, distribution and use of vaccines ${ }^{10-12}$. As noted above, several government agencies are partners in the CVI, a global coalition working to maximize protection against infectious diseases through the development and use of safe, effective, easy-to-deliver and widely available vaccines. Coordination of national malaria vaccine efforts is conducted through the Malaria Vaccine Coordinating Committee, which has developed new collaborations among federal agencies. In addition, programs such as the National Cooperative Vaccine Discovery Group bring together experienced investigators from academia, industry and government to facilitate the conceptualization, discovery and development of HIV vaccines.

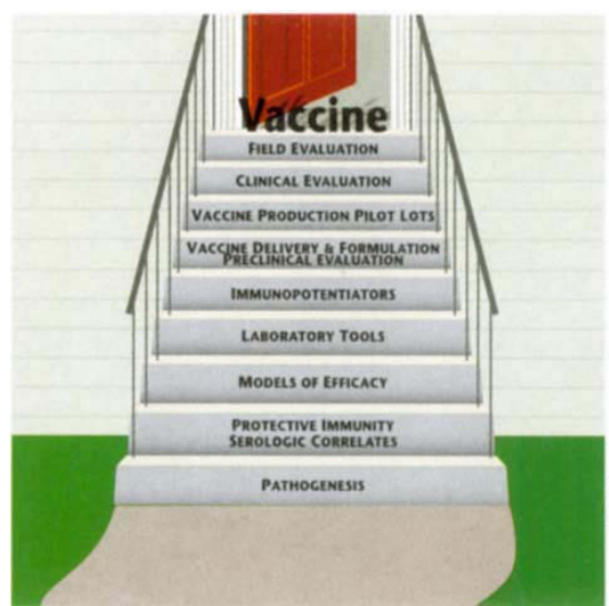

Fig. 3 Steps to vaccine development will provide a useful roadmap for developing vaccines for both infectious and non-infectious diseases such as cancer and autoimmune diseases.

\section{Setting the stage for product development}

$\mathrm{NIH}$ and other agencies actively pursue research portfolios that involve interaction with industry and academia and the transfer of technology to the private sector for commercialization. Historically, an important focus of these efforts has been to further explore concepts that may not be of immediate financial interest, including those for which the principal market might be less developed nations, but nonetheless are of great potential public health importance.

For example, 25 years ago, when very little was known about viruses that cause gastrointestinal diseases, NIH researchers helped identify rotavirus as the leading cause of severe diarrhea in infants and young children ${ }^{1}$. They embarked on the development of a rotavirus vaccine, and after a series of pivotal discoveries developed a rhesus rotavirus-tetravalent (RRV-TV) vaccine to protect against the four clinically important strains of the virus. Having demonstrated the feasibility of this approach, which probably would not have been pursued alone by industry, the NIH team signed a Cooperative Research and Development Agreement (CRADA) with a leading vaccine manufacturer in 1987, which began commercial development of the RRV-TV vaccine. Subsequently, the vaccine has proven safe and efficacious in both developed and developing countries. Widespread use of the RRV-TV vaccine promises to reduce the enormous global burden of rotavirus diarrhea, which claims the lives of an estimated 870,000 children annually ${ }^{19}$ (see Sansonetti, page 499).

The government also plays a critical role in vaccine development by providing scientists with reagents that might not otherwise be shared because of proprietary interests. Of growing importance are repositories such as the AIDS Research and Reference Reagent Program of the NIH, which now stocks over 1,100 reagents for public distribution, including viruses, proteins/peptides, cell lines, recombinant DNA clones and antibodies $^{20}$. Other repositories provide genetically altered mice and research materials relevant to malaria and other diseases. The critical role of such repositories was cogently demonstrated most recently during the Hong Kong outbreak of $\mathrm{H} 5 \mathrm{~N} 1$ avian influenza. Fortuitously, as part of NIH's longstanding research into respiratory These consortia follow leads from basic studies of virology, microbiology, molecular biology, immunology, genetics and structural biology to design vaccine strategies ${ }^{18}$.

Decisions regarding the federal government's vaccine priorities are made on the basis of current morbidity and mortality data, recommendations by advisory groups such as the National Vaccine Advisory Committee, and evaluations made by the Institute of Medicine (IOM) of the National Academy of Sciences. In this regard, a new IOM study on Vaccine Research Priorities for the 21st Century, to be published in 1998, viruses, the specific antisera needed to identify the H5N1 avian influenza strain was available in sufficient quantities from the NIH to quickly develop test kits for detecting the avian influenza virus. $\mathrm{CDC}, \mathrm{WHO}$, the Hong Kong Department of Health and other collaborators used these kits in their extraordinarily successful public health response to the outbreak ${ }^{21}$.

A number of government agencies, including DoD and $\mathrm{NIH}$, support projects to sequence the genomes of medically important pathogens. Sequence information can be used in 
many ways, including identifying antigens to incorporate into vaccines. The success of the first microbe sequencing project - the delineation of the complete Haemophilus influenzae genome in 1995-encouraged the current governmentsponsored efforts to sequence the full genomes of many other pathogens, such as Plasmodium spp., Mycobacterium spp., Chlamydia trachomatis, Vibrio cholerae and Neisseria gonorrhoeae $^{22}$. These sequencing efforts have been facilitated by technologies such as DNA chip technology and microarrays that enable the rapid, simultaneous analysis of tens of thousands of genes and hold particular promise in the development of libraries of immunologically important genes. Among many possible uses, such gene libraries will provide a powerful tool to rapidly identify DNA vaccine candidates.

\section{Successful partnering}

Vaccines are complex products, and the process whereby a vaccine is developed and tested requires many steps (Fig. 3). The various partners in vaccine development bring perspectives, resources and skills that are sometimes unique, but more often overlapping and complementary. Industry provides expertise in product development and manufacturing, while many governmental efforts focus on creating and expanding the scientific base in disciplines that underlie product development ${ }^{10-12}$.

A prototypic example of a successful partnership across sectors is the development of acellular pertussis vaccines ${ }^{23}$. In the 1930 s, the US recorded more than 200,000 cases of pertussis each year. With the development and widespread use of whole-cell pertussis vaccines, morbidity and mortality related to pertussis declined dramatically wherever the vaccines were widely used. However, despite their proven record, concerns about the side effects of whole-cell vaccines resulted in a decrease in their use in many parts of the world, and a concomitant increase in pertussis cases ${ }^{1}$.

These and other concerns stimulated the search for an effective acellular vaccine that would have fewer side effects, thereby removing barriers to the immunization of infants and perhaps adults as well. Basic research in government and university laboratories helped provide the insights that enabled industry to develop candidate vaccines. Phase I and Phase II clinical trials of these products, supported by industry and government, were conducted at academic medical centers. International efficacy trials, funded and overseen by industry and government through CRADA's and other mechanisms, and facilitated by public health officials through intergovernmental channels, helped provide the safety and efficacy data necessary for licensure ${ }^{23}$. These new vaccines are at least as effective, and less reactogenic than "whole-cell" pertussis vaccines. Acellular pertussis vaccines are now recommended for all doses in the childhood immunization schedule in the US ${ }^{24}$. The widespread availability of these well-tolerated vaccines will remove a major disincentive to pertussis immunization in the US and abroad.

The integration of expertise brought to the vaccine development process by various partners will continue to be crucial to dealing with the challenges posed by infectious diseases. As we prepare for the public health challenges of endemic, emerging and re-emerging diseases, it is imperative that a robust commitment to basic research and cross-sector collaboration be maintained. Only with such collaborations can we successfully translate basic research findings and technological advances into improved health through immunization.

As we approach the 21st century there is a growing awareness that we are increasingly living in a global community. The concept of the US government playing a contributory role in the fostering of global health has been the subject of increased attention. This was evident in the justification of the FY 1999 National Institute of Allergy and Infectious Diseases budget before the House Appropriations Subcommittee on the Departments of Labor, Health and Human Services, Education, and Related Agencies ${ }^{25}$. In this regard, the contribution of the federal government to vaccine research and development will be critical.

\section{Acknowledgements}

The authors thank Regina Rabinovich and John La Montagne for helpful discussions.

1. Levine, M.M. et al. eds. New Generation Vaccines, 2nd edition. New York: Marcel Dekker Inc., 1997.

2. Gellin, B. (ed.) The Jordan Report: Accelerated Development of Vaccines. Bethesda, Maryland: National Institute of Allergy and Infectious Diseases, 1998.

3. Children's Vaccine Initiative. The CVI strategic plan: managing opportunity and change: a vision of vaccination for the 21 st century. Geneva, 1997.

4. Centers for Disease Control and Prevention. National, state and urban areas vaccination coverage levels among children aged 19-35 months-United States, July 1996- June 1997. MMWR 47(6), 108-116 (1998).

5. World Health Organization. World Health Report 1997, Geneva.

6. Centers for Disease Control and Prevention. Update: isolation of avian influenza A (H5N1) viruses from humans-Hong Kong, 1997-1998. MMWR 46(52-53), 1245-7 (1998).

7. Centers for Disease Control and Prevention. Reduced susceptibility of Staphylococcus aureus to vancomycin-Japan, 1996. MMWR 46(27), 624-626 (1997).

8. Centers for Disease Control and Prevention. Staphylococcus aureus with reduced susceptibility to vancomycin-United States, 1997. MMWR 46(33), 765-766 (1997)

9. World Health Organization. World malaria situation in 1994. Weekly Epidemiological Record 72, 269-274 (1997).

10. Bloom, BR. The United States needs a national vaccine authority. Science 265 , 1378-80 (1994).

11. Douglas, RG. Fostering partnerships for vaccine development: a delicate fabric. Bull NY Acad Med (Summer) 113-123 (1996).

12. National Vaccine Advisory Committee. United States vaccine research: a delicate fabric of public and private collaboration. Pediatrics 100(6), 1015-1020 (1997).

13. Office of Financial Management, Office of the Director, National Institutes of Health.

14. [Editorial]. Two cheers for the multilateral malaria initiative. Nature; 388(6639), 211 (1997).

15. Tanouye, E. Vaccine business is heating up; drug companies see sales surge. Wall Street journal, Feb. 25, 1998.

16. P.L. 104-113, the National Technology Transfer and Advancement Act of 1995.

17. Jenks, S. Varmus strikes "reasonable pricing" clause in CRADAs. JNCI 87(9), 642 (1995).

18. National Institute of Allergy and Infectious Diseases. HIV/AIDS Research Agenda. Bethesda, Md., (1995).

19. Marwick, C. Rotavirus vaccine a boon to children. JAMA 279(7), 489-490 (1998).

20. National Institute of Allergy and Infectious Diseases. NIH AIDS Research and Reference Reagent Program Catalog. Rockville, Md., 1998.

21. Belshe, RB. Influenza as a zoonosis: how likely is a pandemic? Lancet 351(9101), 460-1 (1998).

22. The Institute for Genomic Research, http://www.tigr.org.

23. Fauci, AS. Biomedical research in an era of unlimited aspirations and limited resources. Lancet 348, 1002-3 (1996).

24. Centers for Disease Control and Prevention. Pertussis vaccination: use of acellular pertussis vaccines among infants and young children. Recommendations of the Advisory Committee on Immunization Practices (ACIP). MMWR 46(RR-7), $1-25(1997)$.

25. Department of Health and Human Services. Fiscal Year 1999 Justification of Estimates for Appropriations Committees, National Institutes of Health, Volume II.

National Institute of Allergy and Infectious Diseases, NIH,

Bethesda, Maryland 